\title{
The sL1CAM in sera of patients with endometrial and ovarian cancers
}

\author{
Michał Wojciechowski ${ }^{1,2}$ (1) $\cdot$ Ewa Głowacka $^{3} \cdot$ Miłosz Wilczyński $^{1} \cdot$ \\ Anna Pękala-Wojciechowska ${ }^{4}$ Andrzej Malinowski ${ }^{1,2}$
}

Received: 13 June 2016/ Accepted: 2 November 2016/Published online: 10 November 2016

(C) The Author(s) 2016. This article is published with open access at Springerlink.com

\begin{abstract}
Purpose L1CAM is a cell adhesion molecule suspected to play an important role in carcinogenesis. The objective of the study was to evaluate the level of soluble L1CAM in the sera of patients with endometrial and ovarian carcinomas and verify the feasibility of the SL1CAM as a marker of these carcinomas.

Methods 35 endometrial and 18 ovarian cancer patients were enrolled in the study. 43 patients with benign gynecological conditions constituted a control group. The sL1CAM serum level was measured with ELISA test in each patient and it was referred to the data from the surgical staging of the cancers.

Results The sL1CAM serum level was significantly lower in patients with endometrial cancer than in healthy women and slightly lower in the ovarian cancer group than in the control group. In the endometrial cancer group there was no correlation between SL1CAM concentration and cancer histopathology, stage or grade. SL1CAM concentration positively correlated with ovarian cancer stage and (not significantly) with grade.
\end{abstract}

Michał Wojciechowski

wojmich@gmail.com

1 Department of Surgical, Endoscopic Gynecology and Oncology, Polish Mother's Memorial Hospital-Research Institute, 281/283 Rzgowska St., 93-338 Łódź, Poland

2 Department of Surgical and Endoscopic Gynecology, Medical University of Lodz, Łódź, Poland

3 Department of Laboratory Diagnostics, Polish Mother's Memorial Hospital-Research Institute, Łódź, Poland

4 Clinical Pharmacology Department, Medical University of Lodz, Łódź, Poland
Conclusions Despite the increasing data about the possible role of L1CAM as a strong prognostic factor of poor outcome in many cancers, we did not find evidence supporting the use of SL1CAM as a marker of endometrial or ovarian cancers.

Keywords SL1CAM - Ovarian carcinoma $\cdot$ Endometrial carcinoma

\section{Introduction}

Endometrial cancer is the most common cancer of the female genital tract in developed countries. Fortunately, it is diagnosed relatively quickly in many patients due to its early symptom-abnormal uterine bleeding. In the majority of cases, the cancer is discovered at FIGO stage I and presents endometrioid morphology (so-called "type 1" endometrial cancer) which can be cured in almost $90 \%$ of patients. About $10 \%$ of these patients, with potentially favorable prognosis, will however relapse and die from the disease. Some patients are diagnosed at more advanced stages or present with "type 2" papillary serous or clear cell endometrial cancer with substantially worse prognosis [1-3]. There is also a certain portion of uterine cancers of mixed morphology where the prognosis is particularly difficult to establish. The management of endometrial cancer consists of preoperative workup (dilatation and curettage, transvaginal ultrasonography, MRI, CT) followed by surgical staging (hysterectomy, bilateral salpingoophorectomy, pelvic lymphadenectomy in patients at high risk of relapse), which is meant to establish the final diagnosis, essential for prognosis and proper treatment [2-9]. These measures, however, are not sufficient to recognize the group of patients with early stage type 1 
endometrial cancer, who, despite favorable prognosis and adequate treatment, will eventually die from the disease. In recent studies, L1CAM has been identified as a possible marker of poor prognosis and relapse in patients with endometrial cancer both of type 1, type 2 and mixed morphology [10-13].

Epithelial ovarian cancer remains one of the most frequent causes of cancer-related deaths in women. Due to lack of symptoms and no screening tests, it is seldom discovered at the early stages; therefore, the outcome is unfavorable in many cases. The management typically consists of primary debulking surgery, followed by platinum-based chemotherapy. Despite the treatment, often with complete response to therapy, the overall 5-year survival rate is disappointing and does not exceed $40 \%$ [14-16]. Therefore, there is a strong need for a marker which could either serve as a screening test and improve early detection or identification of the patients at high risk of chemoresistance and relapse. L1CAM again has been found to be a potential marker of poor outcome, short time to relapse and platinum resistance in ovarian cancer patients $[10,11,17,18]$.

L1CAM is a $200-220 \mathrm{kDa}$ transmembrane adhesion molecule from the immunoglobulin family, consisting of an extracellular portion (six Ig-like domains with five fibronectin-type III repeats), a transmembrane part and a highly conservative cytoplasmatic tail $[10,19,20]$. Originally, it was discovered on neuronal cells and found to play a role in nervous system development [21]. Indeed, in healthy tissues, it is only expressed in collecting tubules in kidneys and peripheral nerve bundles. Hematopoietic cells such as B lymphocytes, $\mathrm{T}$ lymphocytes, and monocytes express rather low levels of L1CAM [11, 22]. It has also been found on the healthy ovarian surface epithelium [23]. It was however reported to be abnormally intensively expressed on many human cancer cells, including endometrial cancer, ovarian cancer, melanoma, colon adenocarcinoma positive to chromogranin, clear cell adenocarcinoma of the urinary bladder, pheochromocytoma, small cell lung carcinoma and tumors of the nervous system, and was identified as a possible marker of advanced stage, invasion and metastasis [22-26]. The regulation of its expression is not well understood and may be influenced by at least several mechanisms (demethylation of L1CAM promoter, TGF- $\beta$ treatment, transcription factor SLUG overexpression or miR-34a expression) [12, 27, 28]. L1CAM can also be detected in its soluble form (sL1CAM) in the serum and ascites fluid from patients with ovarian, uterine and other cancers [10, 19, 24, 29-31]. The process of L1CAM cleavage, mediated by proteases, mainly ADAM10, enhances the ovarian and uterine cancer cell migration on various extracellular matrix components through autocrine/paracrine binding to integrins. This phenomenon may be responsible for accelerated tumor dissemination in L1CAM-positive tumors [10].

The hallmarks of carcinogenesis are progression of the primary tumor and formation of distant metastases, which demand substantial rearrangement in cell and tissue morphology [32]. Cell-to-cell and cell-to-matrix adhesion mediated by adhesive molecules of several families provide not only the structural support of the tissue, but also play an important role in the regulation of many processes such as proliferation, migration, angiogenesis, vascular sprouting and differentiation, which are essential for invasion and metastasis formation [32-38]. Some of the adhesive molecules, like E-cadherin, are responsible for homophilic intercellular interactions and proper tissue structure [39]. Others, like L1CAM, bind the cell to the matrix components during cell migration [12]. Formation of metastasis is believed to begin with loss of E-cadherindependent connections which allows the escape of the cell from its surrounding. It subsequently would migrate along the extracellular matrix components, which is mediated by other adhesive molecules such as CD44 or L1CAM $[12,19,37,38,40,41]$.

\section{L1CAM in endometrial and ovarian cancers}

L1CAM is not expressed in normal endometrium [12, 22]. It had been believed to be absent on the ovarian surface epithelium, stromal cells of the ovary and oocytes until Zecchini et al. found it to be abundant on the ovarian surface epithelium [23]. It however may be highly expressed on the endometrial and ovarian cancer cell surface $[10-12,17,23]$. It is absent in the majority of the early-stage endometrioid endometrial cancer (type 1) cells and is usually strongly expressed on papillary serous and clear cell cancer (type 2) endometrial cells. Its expression negatively correlates with the expression of E-cadherin and estrogen/progesterone receptors, known markers of good prognosis. Thus, L1CAM-negative endometrial cancers tend to be E-cadherin and ER/PR positive [12]. However, there is a certain number of type 1 endometrioid endometrial cancers, positive for L1CAM as well as E-cadherin, but ER/PR negative and thus of type 2-like profile. L1CAM-positive cells may be found in the clear cell/papillary serous foci of the mixed endometrioid/nonendometrioid morphology cancers, which might facilitate identification of such small areas of differentiation within the endometrioid, L1CAM-negative background [12]. In the L1CAM-positive endometrial cancers, like in colon and ovarian cancers, the L1CAM may often be found at the leading edge of the cancer-the area, which also tends to be E-cadherin and ER/PR negative. It has been concluded that such similar and repeatable inverse correlation of 
L1CAM and E-cadherin and ER/PR receptors' expression may suggest their participation in the process of epithelialmesenchymal transition (EMT) [12, 23, 42, 43]. The EMT is a key process during tissue development as well as cancer progression, leading to the acquisition of fibroblastlike morphology of the epithelial cells, reduced intercellular interactions and enhanced motility [42]. As L1CAM is both abundantly present on the normal epithelium of the ovary and the surface of the cells of advanced ovarian cancer, it is suggested to play two opposite roles: in healthy epithelium it would support cell-cell adhesion and apoptosis, whereas in the transformed tissue it would inhibit apoptosis and intercellular interactions, and promote cell proliferation, invasion and transendothelial migration [23].

L1CAM expression is a marker of poor prognosis, short recurrence-free survival and advanced stage of the disease in many cancers including endometrial, ovarian carcinomas, pancreatic ductal adenocarcinoma melanoma and glioblastoma [23, 42]. Although it is not surprising in the case of "type 2" uterine cancers, strikingly, despite potentially good prognosis, type 1 endometrioid cancers, positive for L1CAM, behave in the same manner as type 2 cancers with poor prognosis and short time to recurrence $[10,12,25,26]$. Similarly, in the ovarian cancer, L1CAM tends to be expressed at the advancing edge of the tumor and in all examined patients its expression correlates with high-grade histopathology (G3), advanced FIGO stage, risk of incomplete debulking at primary surgery, lymph node involvement as well as overall and disease-free survival $[10,23,44]$. It is significantly more expressed by cancers with impaired p53 function, which are believed to be more aggressive and resistant to apoptosis and chemotherapy $[18,44]$. What is important, the poor clinical outcome for patients with L1CAM-positive ovarian cancers is similar irrespective of the tumor histological type [10]. Due to its expression being specific to the Mullerian tract-derived cancers like ovarian and endometrial cancer, L1CAM has been suggested as a possible marker differentiating those carcinomas from cases of metastatic cancer of unknown primary site in women [45].

As already mentioned, L1CAM expressed on the surface of cancer cells is released to the body fluids and may be found in serum or ascites fluid of endometrial or ovarian cancer patients, as well as in the culture medium of many human and mouse L1-positive carcinoma cell lines [10, 19, 24, 46]. In ovarian cancer, the L1CAM cleavage intensity is a function of L1CAM surface expression and has been found to be a marker of poor progression-free survival and chemoresistance, although by itself it probably cannot rescue the cells from apoptosis [17, 18, 44]. Several mechanisms are responsible for this phenomenon. One of them is a direct, membrane-proximal cleavage of the extracellular part mainly by ADAM10 protease, which creates $\sim 200 \mathrm{kDa}$ sL1CAM soluble form [19, 24, 46]. The other mechanism, probably predominant in the ovarian cancer, is secretion of sL1CAM in secretory vesiclesexosomes and apoptotic membrane vesicles [24].

It is still unclear whether and to what extent the biological effects of L1CAM are mediated by the soluble form or full-length, membrane-bound particle [42]. The fulllength L1CAM exerts its biological role via several signaling pathways, depending on the substrate attached [42]. The sL1CAM is bound by neurocan-a proteoglycan of the extracellular matrix, which stimulates integrin-mediated cell migration [30, 46] or directly stimulates the cell migration on fibronectin and laminin by autocrine binding to av $\beta 5$ integrin $[19,24,42]$. Thus, sL1CAM promotes cancer progression. It was also found to protect cancer cells from apoptosis in vitro [18, 19]. sL1CAM binds to the integrins on endothelial cells exerting a proangiogenic effect, which is crucial for cancer invasion and may be inhibited by anti-sL1CAM antibodies [29]. The process of sL1CAM shedding has been shown to be involved in the acquisition of chemoresistance by ovarian cancer cells [18] and correlates with progression-free survival (independent prognostic marker) and overall survival of the ovarian cancer patients [44]. There are suggestions that sL1CAM could serve better than Ca125 in the surveillance of free-ofdisease ovarian cancer patients and in searching for recurrence [11]. Such a possible role for SL1CAM as a marker of poor prognosis has been proposed for gastrointestinal stromal tumors [31].

This would suggest that L1CAM expression is invariably implicated in cancer progression-related processes that highly negatively influence the course of the disease. This makes it a potent marker of clinical outcome in ovarian and endometrial cancers, which potentially could modify the diagnostic and therapeutic approach. In a recent multicenter study, L1CAM has been called "the best ever published prognostic factor in FIGO stage I, type I endometrial cancers" [13].

The aim of this study is to assess the concentration of soluble forms of L1CAM in sera of patients with endometrial and ovarian cancer and verify the feasibility of sL1CAM as a marker of the disease and its correlation with clinicopathological parameters of the disease.

\section{Materials and methods}

35 patients with endometrial cancer and 18 with ovarian cancer were operated on in 2013 in the Department of Endoscopic and Surgical Gynecology and Oncological Gynecology, Polish Mother's Memorial Hospital-Research Institute, Łódź, Poland. After the informed consent was obtained, the peripheral blood samples were collected, 
allowed to clot and centrifuged. Serum was stored at $-20{ }^{\circ} \mathrm{C}$. The concentration of soluble forms of L1CAM was assessed with ELISA Uscn E90959Hu set. The clinical data including the histological type of the cancer, the grading and the staging according to the FIGO 2009 were collected after the surgery. These data were matched with the L1CAM serum concentration. Similarly, after the informed consent was obtained, the sera of 43 volunteer patients with benign gynecological conditions were collected for L1CAM soluble form detection. This group served as the control group.

The statistical analysis was made with STATISTICA PL 10 and SPSS 21 software. The distribution of variables was checked with the Shapiro-Wilk test. The qualitative data correlation was verified with Chi-square and Chi-square test with Yates correction. The quantitative data were analyzed with ANOVA Kruskal-Wallis (for three groups) and non-parametrical $U$ Mann-Whitney tests (when two groups were compared). Spearman rank correlation test was used to verify the association between two variables. $p<0.05$ was considered to be significant.

\section{Results}

35 patients with endometrial cancer and 18 patients with cancer of the ovary were included in the study as well as the group of 43 patients with benign gynecological conditions constituting the "control group". The "ovarian cancer" group was significantly younger than the "endometrial cancer" group (54.9 vs. 63.8 years, $p=0.0493$ ) and also younger than the control group (54.9 vs. 62.6 years, $p=0.0682$ ).

The majority of patients with endometrial cancer $(n=22,64.7 \%)$ presented with early stage disease (FIGO I, Table 1) of endometrioid morphology $(n=31,88.6 \%$, Table 2). Most of the patients with ovarian cancer $(n=13$, $72.2 \%$, Table 1) were in advanced stages (at least FIGO III), mainly of papillary serous morphology $(n=9,50.0 \%$, Table 2).

The endometrial cancer was well differentiated in 16 (48.5\%) patients (Table 3) whereas the ovarian cancer was poorly differentiated in $9(50.0 \%)$ patients.

Table 1 FIGO staging of the cancers

\begin{tabular}{llrlll}
\hline Stage & \multicolumn{2}{l}{ Endometrial cancer } & & \multicolumn{2}{c}{ Ovarian cancer } \\
\cline { 2 - 3 } & $N$ & & $N$ & $\%$ \\
\hline 1 & 22 & 64.7 & & 5 & 27.8 \\
2 & 1 & 2.9 & & 0 & 0 \\
3 & 8 & 23.5 & & 13 & 72.2 \\
4 & 3 & 8.8 & & 0 & 0 \\
\hline
\end{tabular}

Table 2 Tumor histopathology

\begin{tabular}{|c|c|c|c|c|}
\hline \multirow[t]{2}{*}{ Histopathology } & \multicolumn{2}{|c|}{$\begin{array}{l}\text { Endometrial } \\
\text { cancer }\end{array}$} & \multicolumn{2}{|c|}{$\begin{array}{l}\text { Ovarian } \\
\text { cancer }\end{array}$} \\
\hline & $N$ & $\%$ & $N$ & $\%$ \\
\hline Endometrioid adenocarcinoma (AE) & 31 & 88.6 & 4 & 22.2 \\
\hline Papillary serous (PS) & 2 & 5.7 & 9 & 50.0 \\
\hline $\mathrm{AE}+\mathrm{PS}$ & 1 & 2.9 & 1 & 5.6 \\
\hline Clear cell $(\mathrm{CC})$ & 0 & 0 & 3 & 16.7 \\
\hline $\mathrm{AE}+\mathrm{CC}$ & 1 & 2.9 & 0 & 0 \\
\hline Anaplastic carcinoma & 0 & 0 & 1 & 5.6 \\
\hline
\end{tabular}

Table 3 Tumor grading

\begin{tabular}{llllll}
\hline Grade & \multicolumn{2}{l}{ Endometrial cancer } & & \multicolumn{2}{c}{ Ovarian cancer } \\
\cline { 2 - 3 } & $N$ & & & $N$ & $\%$ \\
\hline G1 & 16 & 48.5 & & 3 & 16.7 \\
G2 & 12 & 36.4 & & 6 & 33.3 \\
G3 & 5 & 15.2 & & 9 & 50.0 \\
\hline
\end{tabular}

There was an equal distribution of endometrial cancer patients with shallow $(<1 / 2$ of the depth) and deep $(>1 / 2$ of the depth) myometrial invasion (18 vs. 17 patients respectively, ns).

The serum SL1CAM concentration varied significantly between the groups ( $p=0.0062$, Table 4$)$. It was significantly smaller in patients with endometrial cancer than in healthy women ( $p=0.0043)$ and insignificantly smaller in the ovarian cancer group than in the control group $(p=0.603)$.

The results within the groups were, however, importantly spread out which made the analysis very difficult (Fig. 1).

Unfortunately, due to the small groups of patients, it was impossible to verify the correlation between the L1CAM concentration and endometrial or ovarian cancer histopathology. We divided the endometrial cancer patients into "type 1" (endometrioid adenocarcinoma) and "type 2" (non-endometrioid carcinoma) groups and compared them according to the sL1CAM concentration, but the difference turned out to be insignificant. Therefore, the sL1CAM serum concentration did not prove useful in terms of identification of patients with more aggressive, "type 2" cancer morphology (Fig. 2).

In the endometrial cancer group, we have not found any correlation between SL1CAM concentration and tumor stage or grade, or depth of myometrial invasion.

L1CAM concentration positively correlated with ovarian cancer stage ( $p=0.0152, R=0.5618$ ). There is also a positive, but statistically insignificant correlation with 
Table 4 The serum level of L1CAM

\begin{tabular}{lllllllll}
\hline L1CAM & $N$ & Average & Median & Min & Max & Q25 & Q75 & SD \\
\hline Endometrial cancer & 35 & 254.7 & 93.3 & 2.1 & 2652 & 55 & 169 & 505.42 \\
Ovarian cancer & 18 & 474.2 & 117.6 & 2.1 & 3800 & 58 & 289.6 & 953.33 \\
Control group & 43 & 321.4 & 175 & 53 & 1900 & 117 & 227 & 393.18 \\
\hline
\end{tabular}

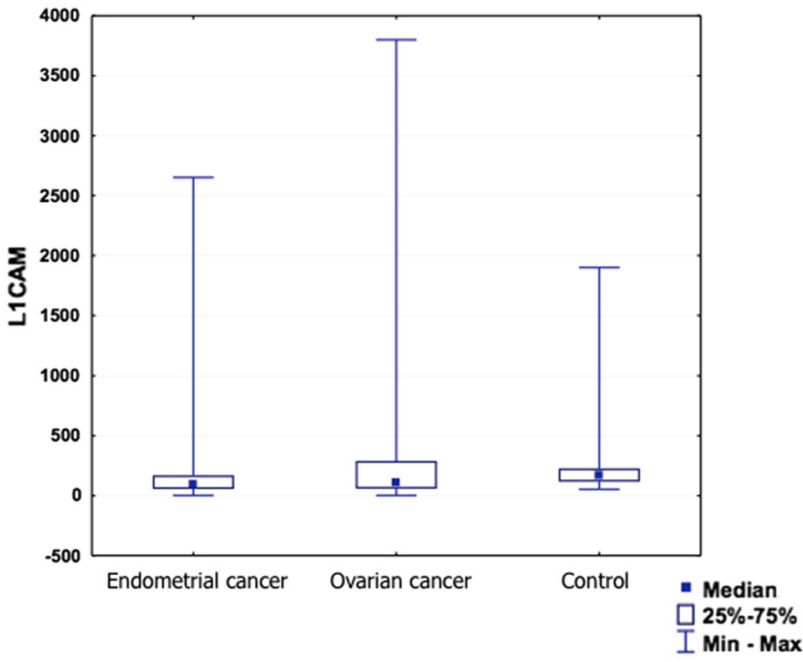

Fig. 1 The serum level of sL1CAM

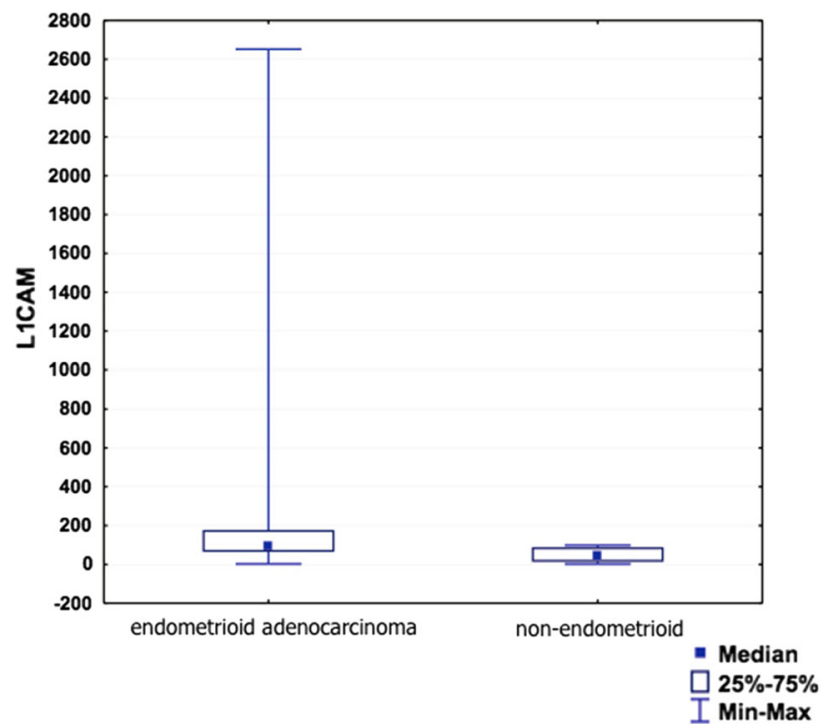

Fig. 2 sL1CAM serum level in type 1 and type 2 endometrial carcinoma patients

ovarian cancer grade $(p=0.0968, R=0.4159)$ (Table 5; Figs. 3,4$)$. Due to a small amount of patients with positive lymph nodes, we were unable to analyze the L1CAM expression in relation to the lymph node status.

We compared the sL1CAM concentration according to distant metastases formation. It turned out to be more expressed in the control group than in cancer patients without metastases (taking both cancers together) $(p<0.01)$. Patients with distant metastases had significantly higher levels of sL1CAM than those without metastases $(p<0.05)$. Analyzing the cancers separately, we found significantly higher levels of sL1CAM in the control group than in endometrial cancer patients without metastases $(p=0.0352)$ and ovarian cancer patients without distant metastases $(p=0.0242)$. Ovarian cancer patients with disseminated disease had higher sL1CAM levels than those without metastases $(p=0.0140)$ (Table 6).

\section{Discussion}

Our investigation is a pilot study, which was supposed to give us an impression on how useful the detection of sL1CAM in serum of patients with uterine or ovarian carcinomas could be. Because it is a prospective study we could not assess the feasibility of SL1CAM as a prognostic factor in terms of survival yet. A vast majority of our endometrial cancer patients had pure endometrioid endometrial cancer (type I) (31 patients out of 35, 88.6\%). Therefore, the type II cancer group was too small (four patients: two non-endometrioid and two mixed morphology) to be evaluated statistically. The sL1CAM concentration was weak and it was even significantly weaker in the sera of uterine cancer patients than in healthy controls group. It is surprising in view of most previously cited data, but as it was already mentioned only $17 \%$ of stage I endometrioid endometrial cancers and up to $28 \%$ of all uterine cancers including type II and advanced stages tumors express L1CAM in their cells $[10,13]$. When we compared the sera of patients with FIGO stage I disease (23 patients) and more advanced stages (12 patients), the sL1CAM level turned out to be insignificantly higher in the letter group (367.1 vs. 200.2, $p=0.51$ ), which could reflect a possible L1CAM positivity in this group. To our knowledge, the percentage of sL1CAM-positive sera among endometrial cancer patients has not been investigated yet. We did not do an immunohistochemical evaluation of cancer specimens for L1CAM membranous expression; thus, we cannot say about the proportion of L1CAM-positive cancers in our group. The same applies to the ovarian cancer group. It has been shown in previous reports that L1CAM is expressed on every ovarian surface epithelium cell, whereas it appears only on a subset of 
Table 5 sL1CAM and endometrial and ovarian cancer stage and grade

\begin{tabular}{lcrl}
\hline & $N$ & Spearman & $p$ \\
\hline L1CAM and endometrial cancer stage & 35 & -0.1540 & 0.3844 \\
L1CAM and endometrial cancer grade & 35 & -0.1137 & 0.5285 \\
L1CAM and ovarian cancer stage & 18 & 0.5618 & 0.0152 \\
L1CAM and ovarian cancer grade & 18 & 0.4159 & 0.0968 \\
\hline
\end{tabular}

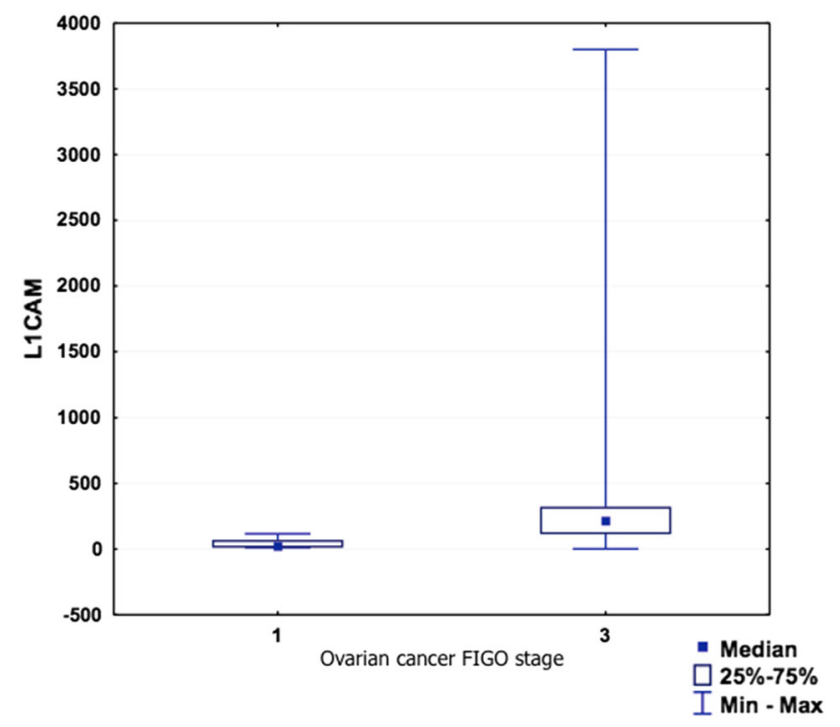

Fig. 3 sL1CAM and ovarian cancer stage

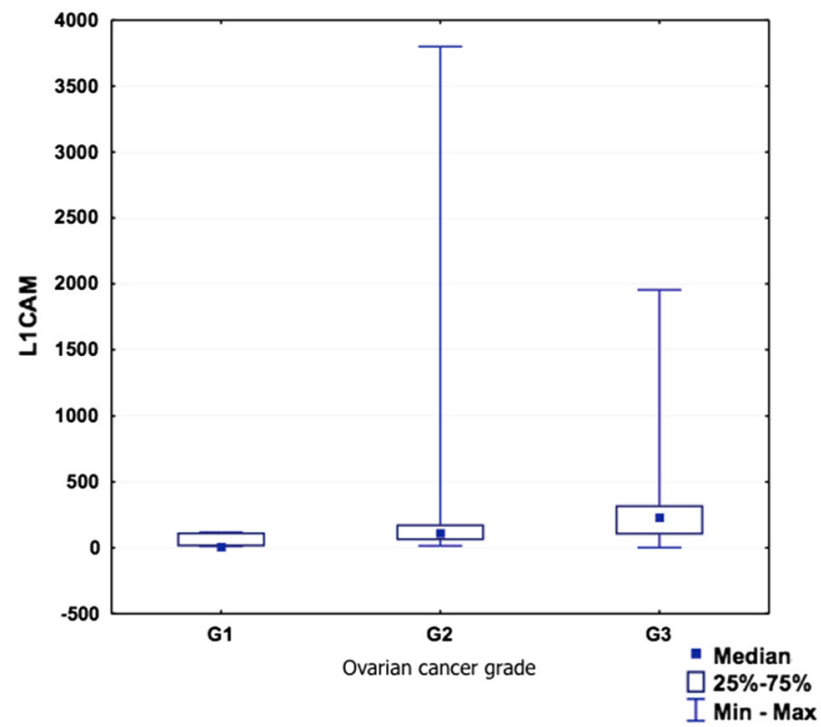

Fig. 4 sL1CAM and ovarian cancer grade

ovarian cancer cells, mainly of advanced stages [23]. To reach conclusive results, a greater number of endometrial cancer patients needs to be investigated for sL1CAM serum level. We therefore could not assess the correlation

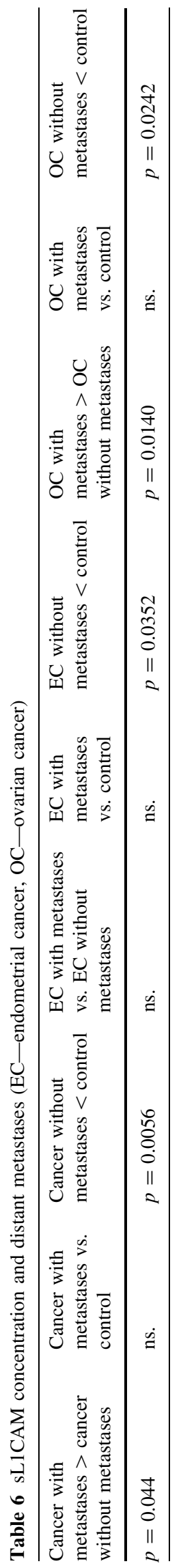


between the L1CAM serum level and endometrial cancer histopathological type, due to a relatively small group of non-endometrioid cancers. There was no correlation of the L1CAM serum level and tumor stage or grade. Again, this could seem surprising in view of recent data suggesting L1CAM to be the most reliable prognostic factor in endometrial cancer. It has to be remembered, however, that these data apply to the membranous expression of the adhesive molecule and have not been analyzed yet in relation to the expression of its soluble forms.

In the ovarian cancer group, the SL1CAM serum level was also slightly lower than in the healthy controls (although not statistically significant, $p=0.608$ ). Although surprising, this result is in line with the report of Zecchini et al. [23]. Moreover, as the sL1CAM may be present in the ascites fluid from ovarian cancer patients via multiple mechanisms, such as direct cleavage by proteases or in secretory vesicles [24], it is possible that in the sera of these patients there also exists a certain heterogeneity of sL1CAM forms. This heterogeneity could affect the sL1CAM detection by the antibodies we used. Among the patients with ovarian cancer, the serum SL1CAM concentration significantly positively correlated $(p=0.015)$ with ovarian cancer FIGO stage, and slightly positively (although without significance, $p=0.096$ ) with tumor grade (G). These results might reflect an increasing L1CAM expression on the surface of ovarian cancer cells in patients with more advanced and aggressive disease [23]. Again, like in the previous group, we have not analyzed the cell membrane expression of L1CAM in ovarian cancer patients and we cannot express the percentage of L1CAM-positive cancers among our patients. As it was mentioned, in ovarian cancer, the L1CAM shedding is a function of its surface expression [44].

Only one patient with endometrial cancer had positive lymph nodes, so that statistic evaluation was impossible. sL1CAM turned out to be more expressed in patients with distant metastases (taking all cancers into account) than without $(p<0.05)$ and in the control group than in the cancer group without metastases $(p<0.01)$. There was no difference between the control group and the cancer group with metastases. The same pattern was observed when the cancers were analyzed separately: both endometrial and ovarian cancer patients without metastases had lower levels of sL1CAM than healthy patients. The level of sL1CAM seemed to rise again when distant metastases appeared, as it was the case in the ovarian cancer group. There were too few patients with endometrial cancer and distant metastases for such analysis. This again might reflect the L1CAM participation in tumor progression and its re-expression and cleavage in the more advanced stages. The most probable explanation is that, according to previous reports, L1CAM cleavage is a function of its surface expression. As it is abundantly expressed on healthy ovarian epithelium and reappears only on advanced ovarian cancer cells, the serum sL1CAM concentration reflects this pattern. The molecule becomes again detectable in patients with advanced disease. If it was the case, sL1CAM could not be an early marker of the disease, but rather a marker of an advanced stage of the cancers.

To conclude, despite the increasing data about the possible role of L1CAM as a strong prognostic factor of poor outcome in many cancers, including endometrial and ovarian cancer as well as the promising data concerning the possibility of detection of L1CAM soluble forms in sera of cancer patients, we did not find evidence for SL1CAM feasibility as a marker of endometrial or ovarian cancers.

We believe a study including more numerous groups of patients could reveal more conclusive results and verify our findings.

Acknowledgements The research was supported by the Polish Mother's Memorial Hospital Research Institute Grant no. 2011/VII/ $13-\mathrm{MN}$.

\section{Compliance with ethical standards}

Conflict of interest We declare that we have no conflict of interest.

Funding All of the study participants are full-time employees of the above-mentioned institution, which founded the research.

Ethical approval All procedures performed in human participants were in accordance with the ethical standards of the institutional and/ or national research committee and with the 1964 Helsinki Declaration and its later amendments or comparable ethical standards. The project was approved by the Ethical Committee of the Polish Mother's Memorial Hospital-Research Institute (Verdict No. 57/2014, 03 July 2014).

Informed consent Informed consent was obtained from all individual participants included in the study.

Open Access This article is distributed under the terms of the Creative Commons Attribution 4.0 International License (http://crea tivecommons.org/licenses/by/4.0/), which permits unrestricted use, distribution, and reproduction in any medium, provided you give appropriate credit to the original author(s) and the source, provide a link to the Creative Commons license, and indicate if changes were made.

\section{References}

1. Brinton LA, Felix AS, McMeekin DS et al (2013) Etiologic heterogeneity in endometrial cancer: evidence from a Gynecologic Oncology Group trial. Gynecol Oncol 129:277-284

2. Creasman WT, Odicino F, Maisonneuve P et al (2006) Carcinoma of the corpus uteri. IJGO 95(Suppl. 1):S105-S143

3. Zaino RJ (2009) FIGO staging of endometrial adenocarcinoma: a critical review and proposal. Int J Gynecol Pathol 28:1-9

4. Amant F, Moerman P, Neven P et al (2005) Endometrial cancer. Lancet 366:491-505 
5. Ambeba E, Linkov F (2001) Advancements in the use of blood tests for cancer screening in women at high risk for endometrial and breast cancer. Future Oncol 7:1399-1414

6. Burke WM, Orr J, Leitao M et al (2014) Endometrial cancer: a review and current management strategies: Part I. Gynecol Oncol 134:385-392

7. Burke WM, Orr J, Leitao M et al (2014) Endometrial cancer: a review and current management strategies: Part II. Gynecol Oncol 134:393-402

8. Mariani A, Sebo TJ, Katzmann JA, Roche PC, Keeney GL, Lesnick TG, Podratz KC (2005) Endometrial cancer: can nodal status be predicted with curettage? Gynecol Oncol 96:594-600

9. Gilani S, Anderson I, Fatallah L et al (2014) Factors predicting nodal metastasis in endometrial cancer. Arch Gynecol Obstet 290:1187-1193

10. Fogel M, Gutwein P, Mechtersheimer S et al (2003) L1 expression as a predictor of progression and survival in patients with uterine and ovarian carcinomas. Lancet 362:869-875

11. Fogel M, Huszar M, Altevogt P, Ben-Arie P (2004) L1 (CD171) as a novel biomarker for ovarian and endometrial carcinomas. Expert Rev Mol Diagn 4:455-462

12. Huszar M, Pfeifer M, Schrimer U et al (2010) Up-regulation of L1CAM is linked to loss of hormone receptors and E-cadherin in aggressive subtypes of endometrial carcinomas. J Pathol 220:551-561

13. Zeimet AG, Reimer D, Huszar M et al (2013) L1CAM in earlystage type- 1 endometrial cancer: results of a large multicenter evaluation. J Natl Cancer Inst 105:1142-1150

14. Heintz APM, Odicino F, Maisonneuve P et al (2006) Carcinoma of the ovary. IJGO 95(Suppl. 1):S161-S192

15. Jayson GC, Kohn EC, Kitchener HC, Ledermann JA (2014) Ovarian cancer. Lancet 384:1376-1388

16. Jelovac D, Armstrong DK (2011) Recent progress in the diagnosis and treatment of ovarian cancer. CA Cancer J Clin 61:183-203

17. Daponte A, Kostopoulu E, Kollia P et al (2008) L1(CAM) (CD171) in ovarian serous neoplasms. Eur J Gynaecol Oncol 29:26-30

18. Stoeck A, Gast D, Sanderson MP et al (2007) L1-CAM in a membrane-bound or soluble form augments protection from apoptosis in ovarian carcinoma cells. Gynecol Oncol 104:461-469

19. Mechtersheimer S, Gutwein P, Agmon-Levin N et al (2001) Ectodomain shedding of L1 adhesion molecule promotes cell migration by autocrine binding to integrins. J Cell Biol 155:661-673

20. Moos M, Tacke R, Scherer H, Teplow D, Fruh K, Schachner M (1988) Neural adhesion molecule L1 as a member of the immunoglobulin superfamily with binding domains similar to fibronectin. Nature 334:701-703

21. Maness PF, Schachner M (2007) Neural recognition molecules of the immunoglobulin superfamily: signaling transducers of axon guidance and neuronal migration. Nat Neurosci 10:19-26

22. Huszar M, Moldenhauer G, Gschwendc V et al (2006) Expression profile analysis in multiple human tumors identifies L1 (CD171) as a molecular marker for differential diagnosis and targeted therapy. Hum Pathol 37:1000-1008

23. Zecchini S, Bianch M, Colombo N et al (2008) The differential role Of L1 in ovarian carcinoma and normal ovarian surface epithelium. Cancer Res 68:1110-1118

24. Gutwein P, Stoeck A, Riedle S et al (2005) Cleavage of L1 in exosomes and apoptotic membrane vesicles released from ovarian carcinoma cells. Clin Cancer Res 11:2492-2501

25. Raveh S, Gavert N, Ben-Ze'ev A (2009) L1 cell adhesion molecule (L1CAM) in invasive tumors. Cancer Lett 282:137-145
26. Schafer MK, Altevogt P (2010) L1CAM malfunction in the nervous system and human carcinomas. Cell Mol Life Sci 67:2425-2437

27. Schirmer U, Fiegl H, Pfeifer M et al (2013) Epigenetic regulation of L1CAM in endometrial carcinoma: comparison to cancer-testis (CT-X) antigens. BMC Cancer 13:156

28. Schrimer U, Doberstein K, Rupp AK et al (2014) Role of miR$34 \mathrm{a}$ as a suppressor of L1CAM in endometrial carcinoma. Oncotarget 5:462-472

29. Friedli A, Fischer E, Novak-Hofer I et al (2009) The soluble form of the cancer-associated L1 cell adhesion molecule is a pro-angiogenic factor. Int J Biochem Cell Biol 41:1572-1580

30. Gutwein P, Oleszewski M, Mechterscheimer S et al (2000) Role of Src kinases in the ADAM-mediated release of L1 adhesion molecule from human tumor cells. J Biol Chem 275:15490-15497

31. Zander H, Rawnaq T, von Wedemeyer M et al (2011) Circulating levels of cell adhesion molecule L1 as a prognostic factor in gastrointestinal stromal tumor patients. BMC Cancer 11:189-196

32. Mareel M, Leroy A (2003) Clinical, cellular, and molecular aspects of cancer invasion. Physiol Rev 83:337-376

33. Aplin AE, Howe A, Alahari SK, Juliano RL (1998) Signal transduction and signal modulation by cell adhesion receptors: the role of integrins, cadherins, immunoglobulin-cell adhesion molecules, and selectins. Pharmacol Rev 50:197-263

34. Golias CH, Chralabopoulos A, Peschos D et al (2005) Adhesion molecules in cancer invasion and metastasis. Hippokratia 9:106-114

35. Pasieka Z (2003) Rola cząsteczek adhezji komórkowej (CAM) w procesie wzrostu i inwazji guzów litych oraz perspektywy ich wykorzystania jako markerów rozrostu nowotworowego w praktyce klinicznej. Onkol Pol 1:39-44

36. Saiki I (1997) Cell adhesion molecules and cancer metastasis. Jpn J Pharmacol 75:215-242

37. Wojciechowski M, Krawczyk T, Śmigielski J et al (2010) The role of adhesive molecules in endometrial cancer: part II. Przegląd Menopauzalny 6:357-361

38. Wojciechowski M, Krawczyk T, Śmigielski J et al (2010) The role of adhesive molecules in endometrial cancer: part I. Przegląd Menopauzalny 5:291-295

39. Frixen UH, Behrens J, Sachs M et al (1991) E-cadherin-mediated cell-cell adhesion prevents invasiveness of human carcinoma cells. J Cell Biol 113:173-185

40. Goodison S, Urquidi V, Tarin D (1999) CD44 cell adhesion molecules. Mol Pathol 52:189-196

41. Shtutman M, Levina E, Ohouo P et al (2006) Cell adhesion molecule L1 disrupts E-cadherin-containing adherens junctions and increases scattering and motility of MCF7 breast carcinoma cells. Cancer Res 66:11370-11380

42. Kiefel H, Bondong S, Hazin J et al (2012) L1CAM. A major driver for tumor cell invasion and motility. Cell Adhes Migr 6:374-384

43. Pfeifer M, Schrimer U, Geisman C et al (2010) L1CAM expression in endometrial carcinomas is regulated by usage of two different promoter regions. BMC Mol Biol 11:64

44. Bondong S, Kiefel H, Hielscher T et al (2012) Prognostic significance of L1CAM in ovarian cancer and its role in constitutive NF- $\kappa B$ activation. Ann Oncol 23:1795-1802

45. Ben-Arie A, Huszar M, Ben-Zvi N et al (2008) The role of L1CAM immunohistochemical staining in the diagnosis of abdominal-pelvic cancer of uncertain primary site in women. EJSO 34:795-799

46. Li Y, Galileo DS (2010) Soluble L1CAM promotes breast cancer cell adhesion and migration in vitro, but not invasion. Cancer Cell Int 10:34 